\title{
Exploring the magnetic fields in local and distant galaxies
}

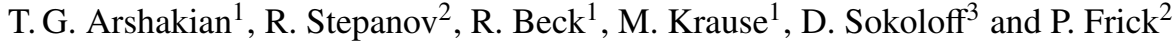 \\ 1 Max-Planck-Institut für Radioastronomie, Auf dem Hügel 69, 53121 Bonn, Germany * \\ e-mail: tigar@mpifr-bonn.mpg.de \\ 2 Institute of Continuous Media Mechanics, Korolyov str. 1, 614061 Perm, Russia \\ 3 Department of Physics, Moscow State University, Moscow 19899, Russia
}

\begin{abstract}
The dominant population of sources at low flux densities observable with future radio telescopes is the population of star-forming disk galaxies which, e.g. with the forthcoming Square Kilometre Array (SKA), would be detected up to about $z \sim 3$ in total radio intensity, and up to $z \sim 5$ with Faraday rotation measures (RM) of intervening disk galaxies towards polarized background quasars. We investigate the possibility to recognize the magnetic field structures in nearby galaxies and to test the cosmological evolution of their large- and small-scale magnetic fields with the SKA and its precursors. We estimate the required density of the background polarized sources detected with the SKA for reliable recognition and reconstruction of magnetic field structures in nearby spiral galaxies. The dynamo theory is applied to distant galaxies to explore the evolution of magnetic fields in distant galaxies in the context of a hierarchical dark matter cosmology. Under favorite conditions, a recognition of large-scale magnetic structures in local star-forming disk galaxies (at a distance $\lesssim 100 \mathrm{Mpc}$ ) is possible from $\gtrsim 10 \mathrm{RMs}$ towards background polarized sources. Galaxies with strong turbulence or small inclination need more polarized sources for a statistically reliable recognition. A reliable reconstruction of the field structure without precognition needs at least $20 \mathrm{RM}$ values on a cut along the projected minor axis which translates to $\approx 1200$ sources towards the galaxy. We demonstrate that early regular fields are already in place at $z \sim 3$ (approximately $1.5 \mathrm{Gyr}$ after the disk formation) in massive gas-rich galaxies $\left(>10^{9} \mathrm{M}_{\odot}\right)$ which then evolve to Milky-Way type galaxies. Major and minor mergers influence the star formation rate and geometry of the disk which has an effect of shifting the generation of regular fields in disks to later epochs. Predictions of the evolutionary model of regular fields, simulations of the evolution of turbulent and large-scale regular fields, total and polarized radio emission of disk galaxies, as well as future observational tests with the SKA are discussed.
\end{abstract}

\section{Introduction}

Most of what we know about galactic magnetic fields comes through the detection of radio waves. Synchrotron emission is related to the total field strength in the sky plane, while its polarization yields the orientation of the regular field in the sky plane and also gives the field's degree of ordering. Incorporating Faraday rotation provides information on the strength and direction of the coherent field component along the line of sight. Faraday rotation measures (RM) in galaxies are generated by regular fields of the galaxy plus its ionized gas, both of which extend to large galactic radii. RM towards polarized background sources can trace regular magnetic fields in these galaxies out to even larger distances, however, with the sensitivity of present-day radio telescopes, the number density of polarized background sources at $1.4 \mathrm{GHz}$ is only a few sources per solid angle of a square degree, so that only M 31 and the LMC could be investigated so far (Han et al. 1998; Gaensler et al. 2005).

The amplitude and structure of magnetic fields in local galaxies is successfully reproduced by the mean-field dynamo theory (Beck et al. 1996). This suggests that the dynamo theory can also be applied for distant galaxies to explore the evolution of magnetic fields at high redshifts. The evolution of magnetic

^ This work was supported by the European Commission Framework Program 6, Project SKADS, Square Kilometre Array Design Studies (SKADS), contract no 011938. fields in galaxies is coupled to the formation and evolution of disk galaxies which are fundamental problems in astronomy. Recent high-resolution numerical simulations of disk formation in galaxies showed that a stable disk could be formed at redshifts $z \sim 5-6$ and even higher (Governato et al. 2004; Mayer et al. 2008). A better understanding of the history of magnetism in young galaxies may help to solve fundamental cosmological questions on the formation and evolution of $\mho$ galaxies (Gaensler et al. 2004; Arshakian et al. 2009).

Future high-sensitivity radio facilities will observe polarized intensity and RM for a huge number of faint radio sources, thus providing a high density background of polarized point sources. This opens the possibility to study in detail the largescale patterns of magnetic fields and their superpositions, thus allowing tests of the dynamo theory for field amplification and its ordering. A major step towards a better understanding of galactic magnetism will be achieved by the SKA (wWw . skatelescope.org) and its precursors (ASKAP, MeerKAT).

\section{Recognition of magnetic field structures in local disk galaxies}

The presence of regular kiloparsec-scale magnetic fields in nearby spiral galaxies makes Faraday rotation an ideal tool for studying the structure of magnetic fields in the disk and halo. With the SKA capabilities RMs of hundred and thousands polarized background sources can be measured behind nearby 

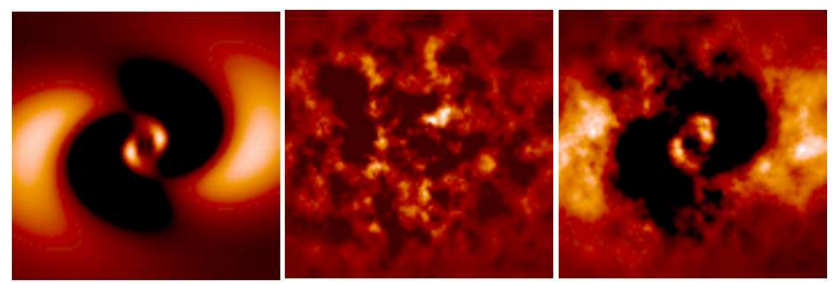

Fig. 1: Left panel: $\mathrm{RM}$ maps (the scale is from +150 to $-150 \mathrm{rad} \mathrm{m}^{-2}$ ) generated for a typical spiral galaxy of radius $10 \mathrm{kpc}$ (the frame size is $20 \times 20 \mathrm{kpc}$ ) with a pure bisymmetric magnetic field structure (regular magnetic field strength of $5 \mu \mathrm{G}$ ) and thermal electron density $n_{0}=0.03 \mathrm{~cm}^{-3}$, at the inclination angle of $i=10^{\circ}$. Middle panel: Modeled random turbulent field for $30 \mathrm{rad} \mathrm{m}^{-2}$ r.m.s. Right panel: In the right panel is the 'realistic' map of RM of the galaxy obtained by superposition of these two field components.

galaxies thus allowing their detailed magnetic field mapping. We estimated the required density of the background polarized sources for reliable recognition and reconstruction of the magnetic field structure in nearby spiral galaxies (Stepanov et al. 2008). For a typical spiral galaxy we modeled the distribution of the ionized gas and the magnetic field in the disk (axisymmetric, bisymmetric and quadrisymmetric spiral, and superpositions). We simulated the RM of a galaxy towards background polarized sources taking into account the RM fluctuations as result of turbulent fields and/or fluctuations in ionized gas (Fig. 1). The simulated magnitudes of RM towards background point sources and their density are then used to recognize the magnetic field structure and assess its reliability.

The slope of the number counts $(\gamma)$ of polarized background sources is unknown beyond $0.5 \mathrm{mJy}$ at $1.4 \mathrm{GHz}$ and important for the field recognition. For the optimistic value of $\gamma=-1.1$, the recognition of a single and mixed modes of regular fields can be reliably performed from a limited sample ( $\gtrsim 10$ ) of RM measurements of polarized point sources. Single higher modes (bisymmetric and quadrisymmetric spirals) are easier to recognize, i.e. they need less RM points, shorter observation time and are less affected by the turbulent component. The dependence on turbulence becomes dramatic for weakly inclined (almost face-on) galaxies - reliable fitting requires a huge number of sources. Future deep (100 h) RM observations with the SKA at $1.4 \mathrm{GHz}$ will allow the recognition of $\lesssim 60000$ spiral galaxies in the volume restricted to a radius of $\sim 100 \mathrm{Mpc}$.

The reconstruction of magnetic field structures of strongly inclined galaxies without precognition of a simple pattern is possible for a large sample ( $>1000)$ of RM point sources. This would require a sensitivity of the SKA at $1.4 \mathrm{GHz}$ of $\approx 0.5-$ $5 \mu \mathrm{Jy}$ (or integration time less than one hour) for galaxies at distances of about $1 \mathrm{Mpc}$. The field structures of $\sim 50$ galaxies until about $10 \mathrm{Mpc}$ distance can be reconstructed with tens to hundred hours of integration time.

Radio telescopes operating at low frequencies (LOFAR, ASKAP, and the low-frequency SKA array) may also be useful instruments for field recognition or reconstruction with the help of RM, if background sources are still significantly polarized at low frequencies. The later reduces the number of RM points per solid angle of the galaxy: (i) the overall degree of polarization is most probably lower due to Faraday depolarization effects, (ii) the typical degree of polarization may decrease for more distant sources (stronger star formation in young galaxies), and (iii) internal and external depolarization may significantly reduce the number of polarized sources. Another problem at low frequencies is thermal absorption that may reduce the radio fluxes at frequencies below about $100 \mathrm{MHz}$ for strongly inclined galaxies with high densities of the ionized gas. For planning surveys at frequencies of $300 \mathrm{MHz}$ and lower, much more needs to be known about the statistics of polarized sources.

\section{Three-phase evolutionary model of magnetic fields in galaxies}

Studying the evolution in magnetic fields of galaxies is important for interpreting future radio synchrotron observations with the SKA. We have used the dynamo theory to derive the timescales of amplification and ordering of magnetic fields in disk and quasi-spherical galaxies (Arshakian et al. 2009). This has provided a useful tool in developing a three-phase evolutionary model of regular magnetic fields, coupled with models describing the formation and evolution of galaxies. In the hierarchical structure formation scenario, we identified three main $\Omega$ phases of magnetic-field evolution in galaxies (Fig. 2). In the epoch of dark matter halo formation (first phase), seed magnetic fields of $\sim 10^{-18} \mathrm{G}$ strength were generated in protogalaxies by the Biermann battery or the Weibel instability (Lazar et al. 2009). Turbulence in the protogalactic halo generated by thermal virialization could have driven the turbulent (smallscale) dynamo and amplified the seed field to the equipartition level of $\approx 20 \mu \mathrm{G}$ within a few $10^{8} \mathrm{yr}$ (second phase). In the epoch of disk formation, the turbulent field served as a seed for the mean-field (large-scale) dynamo developed in the disk (third phase).

- We defined three characteristic timescales for the evolution of galactic magnetic fields: one for the amplification of the seed field, the second one for the amplification of the largescale regular field, and the third one for the coherent field ordering on the galactic scale (Arshakian et al. 2009).

- Galaxies similar to the Milky Way (MW) formed a thick disk at $z \approx 10$, and the mean-field spherical dynamo amplified the field until $z \approx 4$, at which the disk became sufficiently thin, so that the more efficient mean-field disk dynamo could operate and amplified the regular fields within 1.5 Gyr. Regular fields of equipartition (several $\mu \mathrm{G}$ ) strength and a few kpc coherence length were generated within 2 Gyr (until $z \approx 3$ ), but field ordering up to the coherence scale of the galaxy size took another 6 Gyr (until $z \approx 0.5$ ). Giant galaxies (radius $>15 \mathrm{kpc}$ ) had already formed a thin disk at $z \approx 10$, allowing an efficient dynamo generation of equipartition regular fields (with a coherence length of about $1 \mathrm{kpc}$ ) until $z \approx 4$. However, the Universe is too young for fully coherent fields to have already developed in disks of giant galaxies. Dwarf galaxies (radius $<3 \mathrm{kpc}$ ) formed even earlier. If their rotation is ordered and 

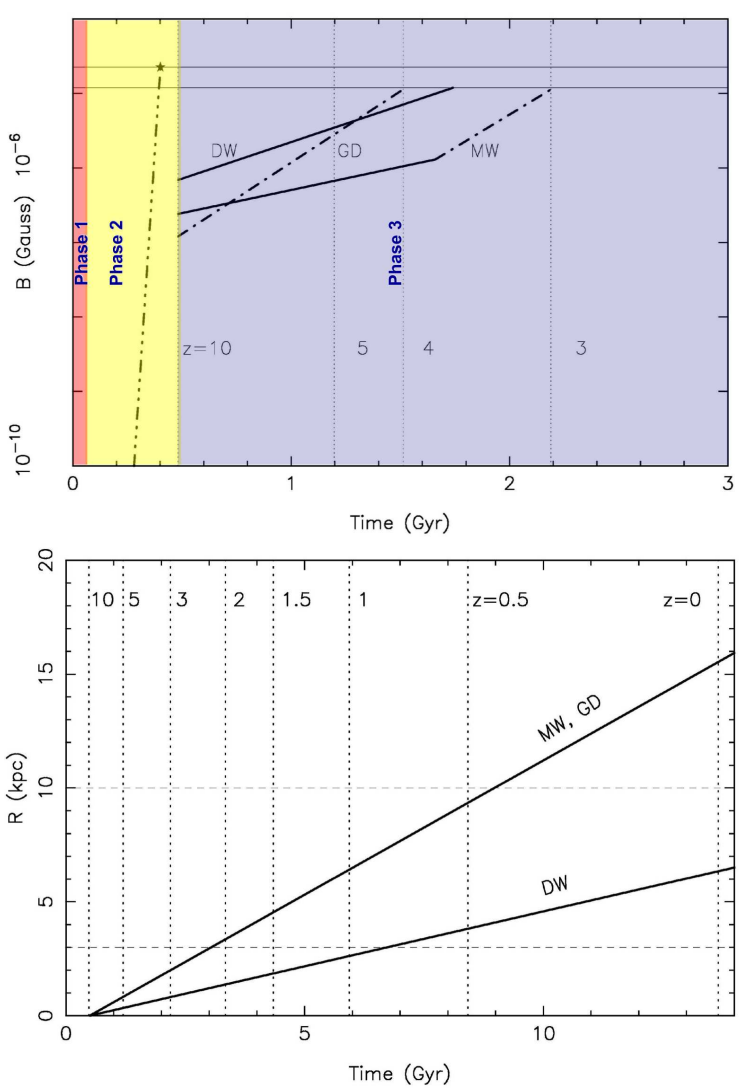

Fig. 2: Top panel: Evolution of magnetic field strength in dwarf galaxies (DW), MW-type galaxies and in giant disk (GD) galaxies. Phase 1 (red): Origin of seed magnetic fields. Phase 2 (yellow): Evolution of the small-scale magnetic field generated by the turbulent dynamo (thick dashed-dot-dot line). Phase 3 (blue): Evolution of the largescale magnetic field generated by the mean-field dynamo in quasispherical galaxies (thick solid line) or in thin-disk galaxies (thick dashed-dot-dashed). At $z \approx 4$ the dynamo type in Milky-Way type galaxies switched from spherical to thin-disk. Bottom panel: Evolution of the ordering scale of regular magnetic fields for dwarf galaxies (DW; bottom line) and disk galaxies (MW and GD; top line).

sufficiently fast to allow the action of the spherical meanfield dynamo, they should have hosted fully coherent fields at $z \approx 1$.

- Major mergers excited starbursts with enhanced turbulence, which in turn amplified the turbulent field, whereas the regular field was disrupted and required several Gyr to recover. Measurement of regular fields can serve as a clock for measuring the time since the last starburst or merger event.

- Starbursts due to major mergers enhance the turbulent field strength by a factor of a few and drive a fast wind outflow, which magnetizes the intergalactic medium. Observations of the radio emission from distant starburst galaxies can provide an estimate of the total magnetic-field strength in the IGM.
We then simulated the evolution of magnetic fields for disk galaxies (Arshakian et al., in prep.) using the timescales of amplification and ordering of regular magnetic fields derived in Arshakian et al. (2009). For a MW-type galaxy ( $R=10 \mathrm{kpc})$, we started from seed fields generated in 0.5 Gyr after the disk formation at $z=10$. The size of the seed $(1 \mathrm{kpc})$, amplitude of its regular field (distributed normally around $0.3 \mu \mathrm{G}$ ) and pitch angle (scattered within $\pm 20^{\circ}$ ) are used to simulate the evolution of regular fields to later epochs (Fig. 3). After 5 Gyr the regular field is amplified to the equipartition level while the field is ordered at scales of $5 \mathrm{kpc}$. After $10 \mathrm{Gyr}$ the field is fully coherent. At low frequencies (150 MHz), significant depolarization caused by regular fields (Fig. 3; bottom panels) results in an asymmetric polarized structure elongated near the minor axis of a galaxy.

\section{Future observational tests with the SKA}

Our analysis has important implications on the expectations of the future observations of magnetic fields with the SKA:The total magnetic field can be measured by the observed total power radio emission, corrected for the thermal fraction of a galaxy, while the regular magnetic field can be traced by polarized synchrotron emission and by Faraday rotation. The tight radio - far-infrared correlation in galaxies implies that radio synchrotron emission is an excellent tracer of star formation in galaxies, at least to distances of $z \simeq 3$ (Seymour et al. 2008).

The small-scale dynamo, with the help of virial turbulence, could have amplified turbulent fields to the level of equipartition with turbulent energy density within $\simeq 3 \times 10^{8}$ years (Arshakian et al. 2009); strong fields should therefore existed in all star-forming galaxies at $z \simeq 10$ (Fig. 2; top panel) and the radio-far-infrared correlation should be valid for $z \lesssim 10$. However, if the total field strength was smaller than $3.25 \mu \mathrm{G}(1+\mathrm{z})^{2}$, the strong cosmic microwave background $(\mathrm{CMB})$ radiation at high redshifts would have suppressed the non-thermal continuum emission of a galaxy by means of inverse Compton losses of cosmic-ray electrons, suggesting that the radio-far-infrared correlation should evolve with infrared/radio ratios increasing with redshift (Murphy 2009). Suppression of the non-thermal component at high redshifts $(z>3)$ would have left only radiation in the thermal (freefree) regime, thus limiting the depth to which the SKA can detect star-forming galaxies. Deviations from nominal IR/radio ratios at high-z will provide a means for constraining the presence and strength of magnetic fields in young galaxies (Murphy 2009). The SKA and its precursor telescopes will investigate this relation in more detail.

A number of predictions of the evolutionary model of magnetic fields can be tested with the SKA's measurements of polarized synchrotron emission and Faraday rotation of distant galaxies: (i) an anticorrelation at fixed redshift between galaxy size and the ratio between ordering scale and the galaxy size, (ii) giant galaxies (radius $>15 \mathrm{kpc}$ ) should not host fully coherent fields (no large-scale RM patterns) until the present epoch, (iii) Milky-Way type galaxies should host fully coherent fields (large-scale RM patterns) at $z \lesssim 0.5$, (iv) undisturbed dwarf galaxies with ordered rotation should host fully coherent fields 


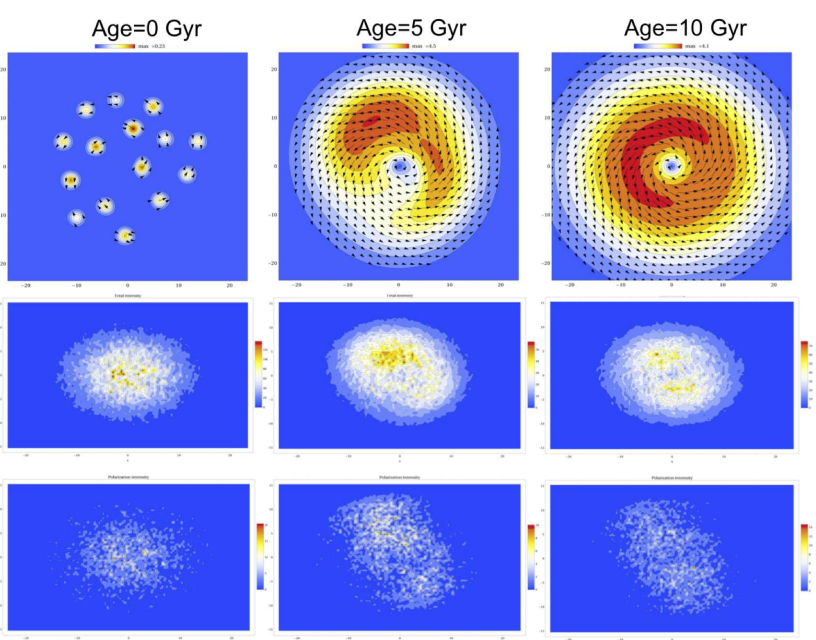

Fig. 3: Simulations (in the rest frame of a galaxy) in the framework of the SKA design studies. First row: the evolution of regular magnetic fields in the disk of a galaxy seen face-on (the frame size is $20 \mathrm{kpc} \times 20 \mathrm{kpc}$ ). The amplitude and ordering scale of the regular fields in $0.5 \mathrm{Gyr}$ after the epoch of disk formation $(0.3 \mu \mathrm{G}$ and $1 \mathrm{kpc}$; left panel), after $5 \mathrm{Gyr}(\sim 1.5 \mu \mathrm{G}$ and $6 \mathrm{kpc}$; middle panel), and after $10 \mathrm{Gyr}(\sim 1.5 \mu \mathrm{G}$ and $12 \mathrm{kpc}$; right panel). Simulations of the total intensity (second row) and polarization (third row) at $150 \mathrm{MHz}$ for a galaxy with an inclination angle of 60 degrees, turbulent $(3 \mu \mathrm{G})$ and regular $(1.5 \mu \mathrm{G})$ magnetic fields, and a star-formation rate of $10 \mathrm{M}_{\odot}$ $\mathrm{yr}^{-1}$ are shown for the same epochs.

giving rise to strong Faraday rotation signals (large-scale RM patterns) at $z \lesssim 1$, and (v) weak regular fields (small Faraday rotation) in galaxies at $z \lessgtr 3$, possibly associated with strong anisotropic fields (strong polarized emission), would be signatures of major mergers (Arshakian et al. 2009).

The method of observing RM against distant polarized background sources can also be applied to measure the strength of the regular field in distant intervening star-forming galaxies, identified by additional optical spectroscopic observations with Mg II absorption systems (Bernet et al. 2008). Deeper observations with future telescopes are essential to provide RM data with much better statistics to larger redshifts $(z \lesssim 5)$ and in much smaller redshift bins and would allow to study the 'RM function' which we define as the number of background polarized sources per RM and redshift intervals. This will allow the evolution of the amplitude and ordering scale of regular fields to be tested (Arshakian et al., in prep.).

In the hierarchical merger formation model, massive galaxies with masses greater than the MW and high star-formation rate $\left(>100 \mathrm{M}_{\odot}\right)$ formed at $z \sim(2$ to 3$)$. On the other hand, observations of undisturbed massive galaxies at earlier epochs support the alternative idea of their formation by accretion of narrow streams of cold gas, as evident from cosmological simulations (Dekel et al. 2009). Polarization observations of massive distant galaxies with the SKA will be crucial to distinguish between different cosmological scenarios of formation and evolution of galaxies.
Another powerful tool to study the magnetic field strength in distant galaxies is the observing of RM against gravitational lens systems. The difference of Faraday rotations of a lens system is attributed mainly to the conditions of the magneto-ionic medium of a lens galaxy, and it is free of intrinsic Faraday rotation of a lens system, Faraday rotation caused by the MilkyWay and intergalactic medium. The Faraday rotation measurements of lens systems can effectively probe the existence of large-scale regular fields in distant star-forming and elliptical galaxies (Narasimha \& Chitre 2004). The SKA with the sensitivity down to an rms of $1 \mu \mathrm{Jy}$ will be able to find few thousands of lens systems per square degree with Einstein radii between $0.5^{\prime \prime}$ and $2^{\prime \prime}$ typical of galaxy lensing (Koopmans et al. 2004). Most of these sources are expected to be star-forming galaxies and radio-quiet active galactic nuclei, thus, allowing to probe the cosmological evolution of magnetic fields in these galax ies beyond $z>1$. In addition, polarized background sources that are lensed into extended arcs can be used, through Faraday rotation, to map RM in the lens itself.

Acknowledgements. TGA acknowledges support from the DFG-SPP project under grant 566960 . This work is also supported by the DFGRFBR project under grant 08-02-92881.

\section{References}

Arshakian T. G., Beck, R., Krause, M., et al. 2009, A\&A, 494, 21

Beck R., et al. 1996, ARA\&A, 34, 155

Beck R. 2005, in Cosmic Magnetic Fields, eds. R. Wielebinski \& R. Beck, Springer, Berlin, p. 43

Bernet M. L., et al. 2008, Nature, 454, 302

Dekel A., et al. 2009, Nature, 457, 451

Gaensler B. M., Beck, R. \& Feretti, L. 2004, New Astr. Rev. 48, 1003

Gaensler B. M., Haverkorn, M., Staveley-Smith, L., et al. 2005, Science, 307, 1610

Governato, F., Mayer, L., Wadsley, J., et al. 2004, ApJ, 607, 688

Han J. L., Beck, R. \& Berkhuijsen, E. M. 1998, A\&A 335, 1117

Koopmans, L. V. E., Browne, I. W. A., \& Jackson, N. J. 2004, New Astronomy Review, 48, 1085

Kronberg P. P., Bernet, M. L., Miniati, F., et al. 2008, ApJ, 676, 70

Lazar M., Schlickeiser, R., Wielebinski, R. \& Poedts, S. 2009, ApJ, 693, 1133

Mayer L., Governato, F. \& Kaufmann, T. 2008, arXiv:0801.3845

Murphy E. J. 2009, ApJ, 706, 482

Narasimha, D., \& Chitre, S. M. 2004, Journal of Korean Astronomical Society, 37, 355

Seymour N., Dwelly, T., Moss, D., et al. 2008, MNRAS, 386, 1695

Stepanov R., Arshakian, T. G., Beck, R., et al. 2008, A\&A, 480, 45 